\title{
Les pompes à volute en béton Bergeron - Applications aux grands transferts d'eau
}

\author{
Bergeron concrete volute pumps - Applications of large water transfers
}

par K. Deschamp, J.F. Lapray, P. Olivier

Alstom ACB Etablissement BERGERON

The concrete volute pump is a well known and reliable way to ensure pumping of large amounts of water in a flow rate range from 4 to $40 \mathrm{~m}^{3} / \mathrm{s}$ with total head from 5 to $35 \mathrm{~m}$ and up to $55 \mathrm{~m}$ with special features.

In a first part the paper deals with a description of the concrete volute pump, integration in pumping station and with various motor/gearbox - pump set arrangement.

In a second part some new developments in progress are described : performance improvement, main components optimization and increasing of the whole pump set reliability.

\section{TECHNOLOGIE DE LA POMPE À VOLUTE EN BÉTON}

La pompe à volute en béton differre des pompes conventionnelles à volute métallique du fait que le conduit d'aspiration et la volute sont construits en béton armé et font partie intégrante de la structure de la station de pompage : la figure 1 représente les principaux composants de cette pompe. On

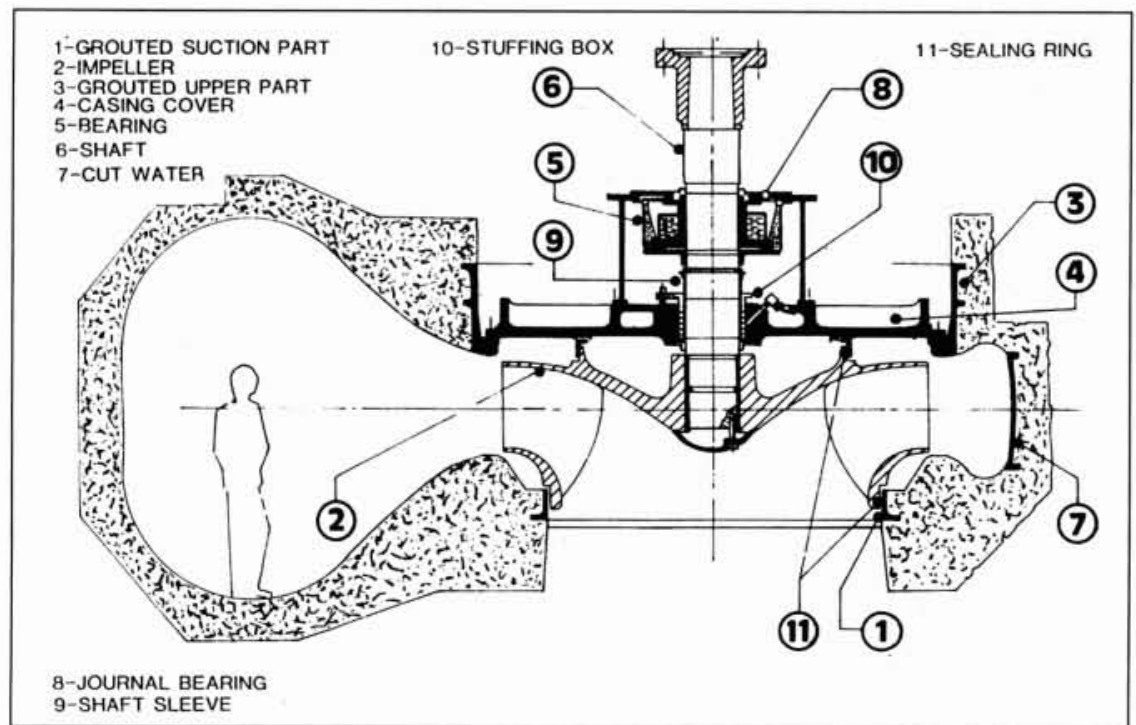

1. Coupe longitudinale de la pompe à volute en béton. remarque que cette pompe est du type " pull out ", c'est-àdire que l'ensemble des parties métalliques, y compris le fonds de corps, peuvent être démontées en un seul bloc. Les seules parties métalliques statoriques sont les 2 pièces scellées dans le béton et la languette, appelée également " bec de volute " dont le rôle est de protéger les bétons dans cette zone où l'écoulement est le plus turbulent avec les plus grandes pointes de vitesse.

\subsection{Eléments en béton}

\section{On distingue :}

- le conduit d'aspiration (fig. 2).

Il est inséré dans le Génie Civil de la station de pompage. Différentes formes peuvent être adoptées de façon à satisfaire aux contraintes suivantes :

- forme de la section d'entrée adaptée à la section de sortie du système de filtration,

- perte de charge aussi réduite que possible pour atteindre un haut rendement global de pompage,

- distribution de vitesse quasi-uniforme à l'entrée de la roue pour limiter les effets secondaires de cavitation, bruit, vibrations et perte de rendement,

- niveau bas du conduit d'aspiration aussi peu profond que possible, pour 


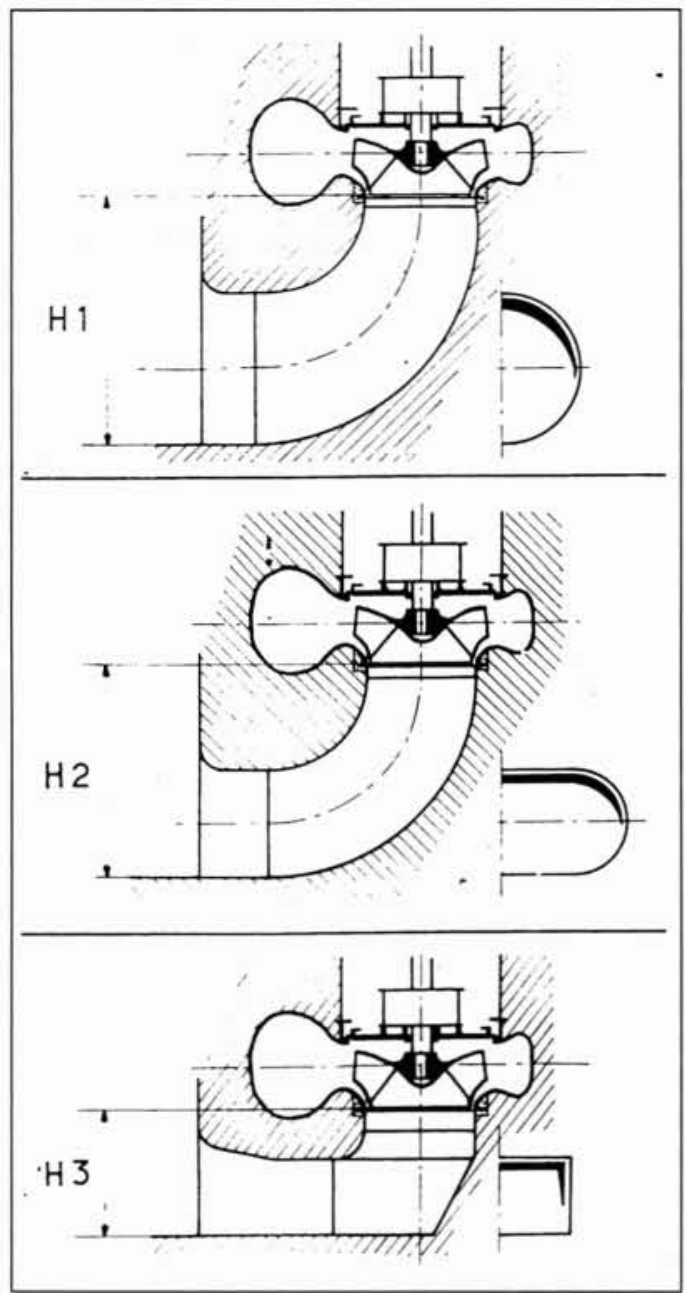

2. Conduits d'aspiration.

limiter le volume des fouilles. La figure 2 montre trois types de conduits d'aspiration correspondant à diverses applications.

- la volute (fig. 3 ).

La forme de la volute est étudiée de façon à répondre à trois exigences :

- un rendement hydraulique le plus élevé possible,

- un coffrage simple à mettre en œuvre,

- une mise en place du ferraillage et une coulée du béton aussi aisées que possible.

La figure 3 donne des exemples des diverses formes de volutes utilisées selon les cas. La volute à double courbure, plus conventionnelle, conduit à un rendement légèrement supérieur et permet, dans certains cas, un raccord plus facile avec les tuyauteries circulaires installées au refoulement de la pompe. Les volutes à sections polygonales utilisent un ferraillage plus simple et des coffrages d'un coût plus réduit et leur raccordement à des galeries carrées ou rectangulaires est également plus facile. Généralement, des roues purement centrifuges sont montées dans les volutes qui possèdent un plan de symétrie. Les roues hélico- centrifuges conviennent mieux aux volutes à sections polygonales ayant un fond plat.

Les parties scellées dans le béton, telles qu'on peut les voir sur la figure 1, comprennent :

- une pièce scellée à l'aspiration, servant à fixer la bague d'étanchéité à l'aspiration de la pompe,

- une pièce scellée en partie supérieure, dont le rôle est de recevoir le fond de corps de pompe,

- une languette scellée, appelée également " bec de volute " dont les dimensions dépendent de la hauteur d'élévation assurée par la pompe. L'un des critères qui permettent de dimensionner cette pièce est la vitesse maximale de l'eau acceptable pour éviter tout risque d'érosion du béton. Elle sert localement au blindage de la volute.

\subsection{Parties démontables (fig. 4)}

L'ensemble des parties démontables est bien mis en évidence sur l'écorché d'une pompe de $24 \mathrm{~m}^{3} / \mathrm{s}$ à $15 \mathrm{~m}$.

L'arbre n'est jamais en contact direct avec l'eau pompée, des chemises sont montées pour protéger l'arbre au droit de l'accouplement et du presse-étoupe. Les paliers sont hors d'eau. En général le palier inférieur est du type à cuve tournante auto-lubrifiée, ce qui assure son autonomie dans tous les cas de fonctionnement, même en cas de rotation inverse. La butée est conventionnelle et peut être installée, soit dans le moteur ou le réducteur, soit directement sur la pompe.

Dans la plupart des cas, l'étanchéité au pompage de l'arbre est assurée au moyen d'un boîtier de presse-étoupe, dans des cas plus rares par une garniture mécanique, souvent refroidis avec l'eau pompée. Dans les cas d'eau chargée, un système de filtration est prévu.

\subsection{Construction et montage}

Le conduit d'aspiration et la volute sont coulés in situ à l'aide de coffrage. Ces derniers sont composés d'éléments en bois assemblés sur site éventuellement réutilisables plusieurs fois. Le béton est de qualité standard ne nécessitant aucun adjuvant ou traitement spécial. Après décoffrage, les surfaces sont laissées brutes et aucun traitement n'est nécessaire même en cas de pompage d'eau de mer comportant des teneurs significatives en sable et en silt. Les coffrages en bois donnent en effet des bétons dont l'état de surface ne nécessite aucune reprise ni traitement (fig. 5).

\section{II - ARCHITECTURE DES STATIONS DE POMPAGE AVEC POMPE À VOLUTE BÉTON}

Ce type de pompe présente une grande souplesse d'adaptation et de flexibilité pour s'adapter aux nombreuses contraintes variables d'un site à l'autre :

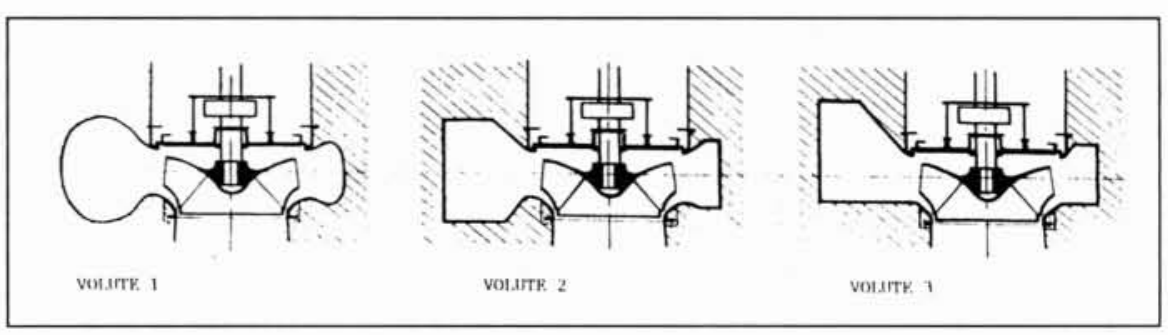

3. Diverses pompes de volute en béton. 


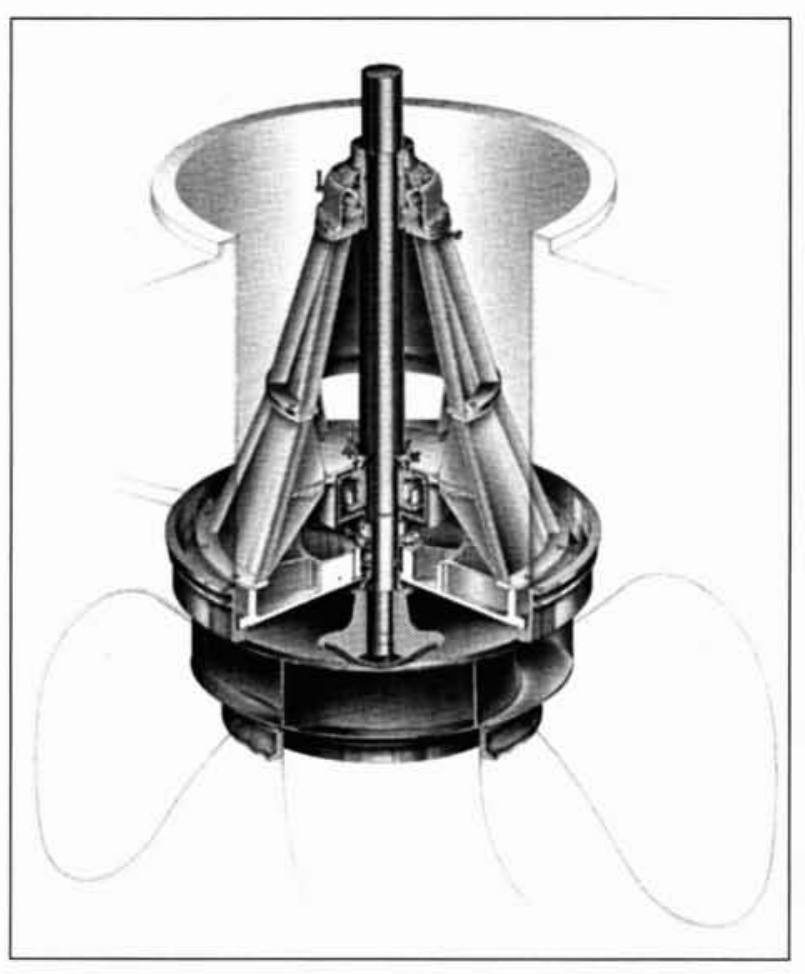

4. Ecorché de la pompe volute béton.

- niveau du radier et niveaux des plus hautes et plus basses eaux,

- raccordement du coude d'aspiration aux systèmes de filtration. Ces derniers, systématiques dans le cas de pompage d'eau de mer, sont également rencontrés dans certaines conditions pour les eaux douces.

Que les systèmes de filtration soient du type Tambour Filtrant ou Filtre Rotatif pour alimenter une ou deux pompes, la liaison filtre - conduit d'aspiration doit respecter les règles de base conduisant, dans tous les cas de fonctionnement, à une alimentation de la pompe sans vortex, ni entrée d'air, avec un écoulement homogène. C'est un des points clefs de la fiabilité de la pompe à volute béton.

Dans les cas les plus délicats, il est judicieux de procéder à un essai sur modèle réduit, en similitude de Froude, pour vérifier avant la construction de la station, que les vortex, instabilité, entraînement d'air, zone d'eau morte, ne perturbent pas l'écoulement à l'entrée de la pompe.

La fig. 6 montre quelques principes d'installation d'une pompe à volute béton avec son système de filtration.

Au refoulement, on peut installer des vannes papillon dans le cas où deux pompes refoulent sur une même conduite. Dans cette configuration, une chambre de vanne est prévue avec manchette scellée dans le conduit de refoulement.

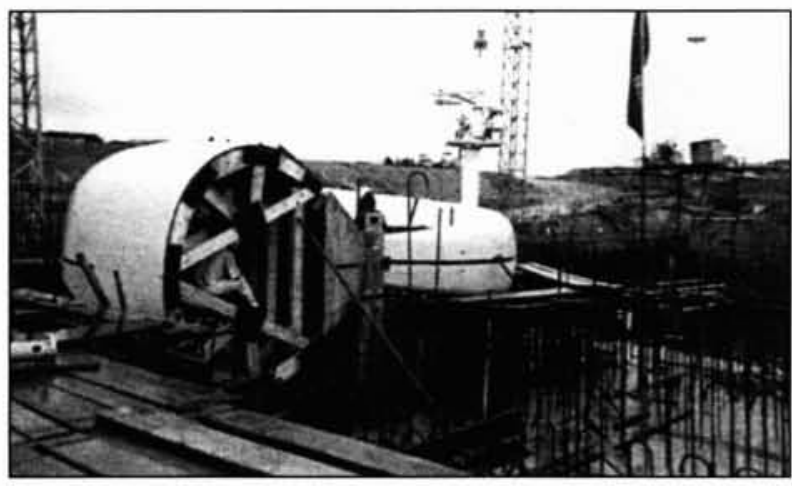

5. Coffrage bois de pompe de circulation. Centrale de Ling Ao. Débit $24 \mathrm{~m}^{3} / \mathrm{s}$.

De nombreuses pompes à volute béton, utilisées pour le refroidissement des condenseurs des centrales thermiques et/ou nucléaires, ne possèdent pas de vanne au refoulement. Cela est le cas particulièrement pour la quasi totalité du parc de centrales thermiques et nucléaires d'Electricité de France. Dans ces conditions, la pompe doit être conçue pour s'adapter à tous les régimes transitoires (démarrage, arrêt normal et/ou accidentel).

Une étude très poussée du comportement du circuit de circulation doit être effectuée pour assurer la protection des tuyauteries et du condenseur contre les surpressions et les dépressions lors de ces régimes transitoires. Des points particuliers comme le premier remplissage, l'amorçage des boîtes à eau du condenseur, la mise en place des dispositifs de protection (vanne casse-vide, soupape d'entrée d'air, etc.) font l'objet d'attention particulière.

L'expérience acquise aujourd'hui permet de refroidir en eau de mer une centrale nucléaire PWR de $900 \mathrm{MW}$ ou 1300 MW avec 2 pompes alimentées chacune par un tambour filtrant, sans vanne.

Pour la station d'irrigation, il est souvent avantageux d'installer un siphon au refoulement de la pompe à volute béton, eu égard aux faibles hauteurs d'élévation de ces pompes. Là encore, la fiabilité est excellente puisque le seul organe de sûreté consiste en la vanne casse-vide installée en partie haute du siphon.

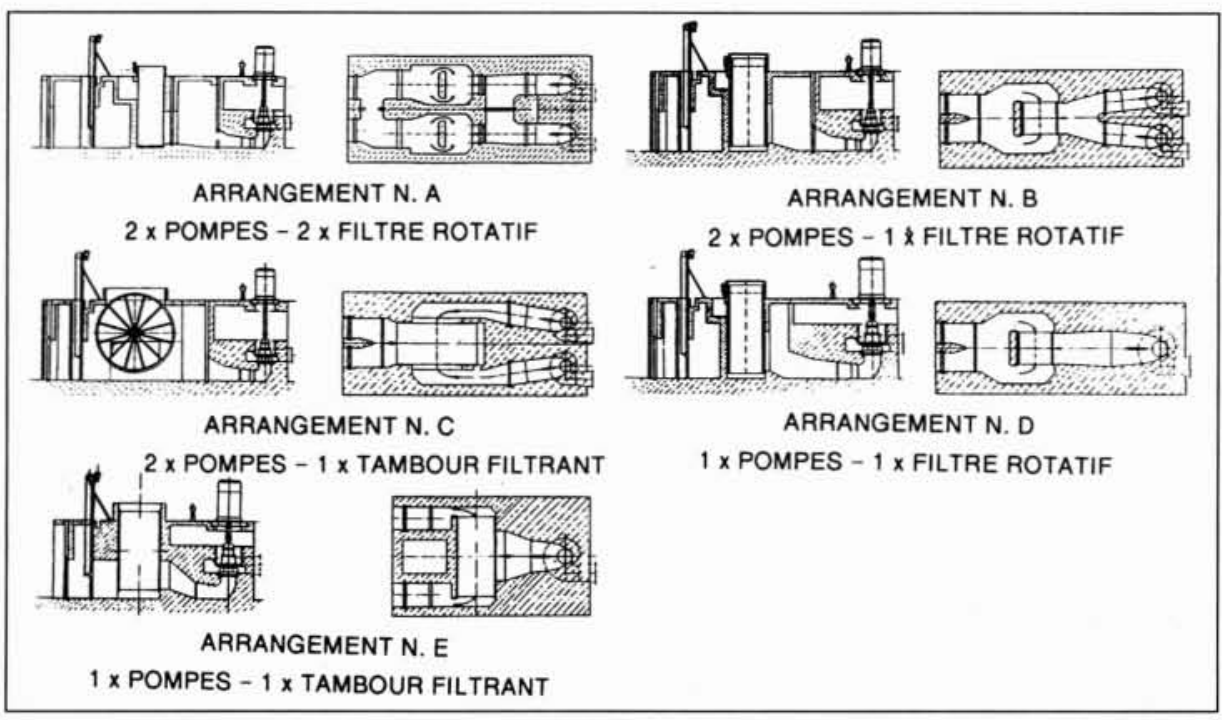

6. Principe d'installation d'une pompe à volute béton avec son système de filtration. 


\section{III — ENTRAÎNEMENT DES POMPES À VOLUTE BÉTON}

L'entraînement des pompes à volute béton dont les vitesses de rotation sont, en général, inférieures à $300 \mathrm{tr} / \mathrm{mn}$ pour des puissances de 1000 à $8000 \mathrm{~kW}$ est aujourd'hui réalisé de deux façons :

- soit par un moteur lent à attaque directe,

- soit par un réducteur entraîné par un moteur rapide.

Le choix du système est essentiellement guidé par deux considérations :

- le coût de l'investissement,

- la fiabilité de fonctionnement et le coût de la maintenance.

Les questions de rendement jouent un rôle plus secondaire car, si le rendement d'un moteur lent est légèrement plus faible que celui du moteur rapide, le rendement du réducteur pénalise le rendement de l'ensemble moteur-réducteur. Les deux solutions sont alors presque comparables sur ce point. Le coût d'un moteur lent est toujours plus élevé que celui d'un groupe moto-réducteur ; par contre il est prouvé que la fiabilité et les coûts de maintenance sont en faveur de la solution à attaque directe.

Dans l'irrigation les problèmes sont différents. Le nombre élevé de groupes, souvent supérieur à 5 en parallèle, et le temps d'utilisation annuel réduit plaident en faveur de la solution avec réducteur conduisant à un investissement initial plus faible.

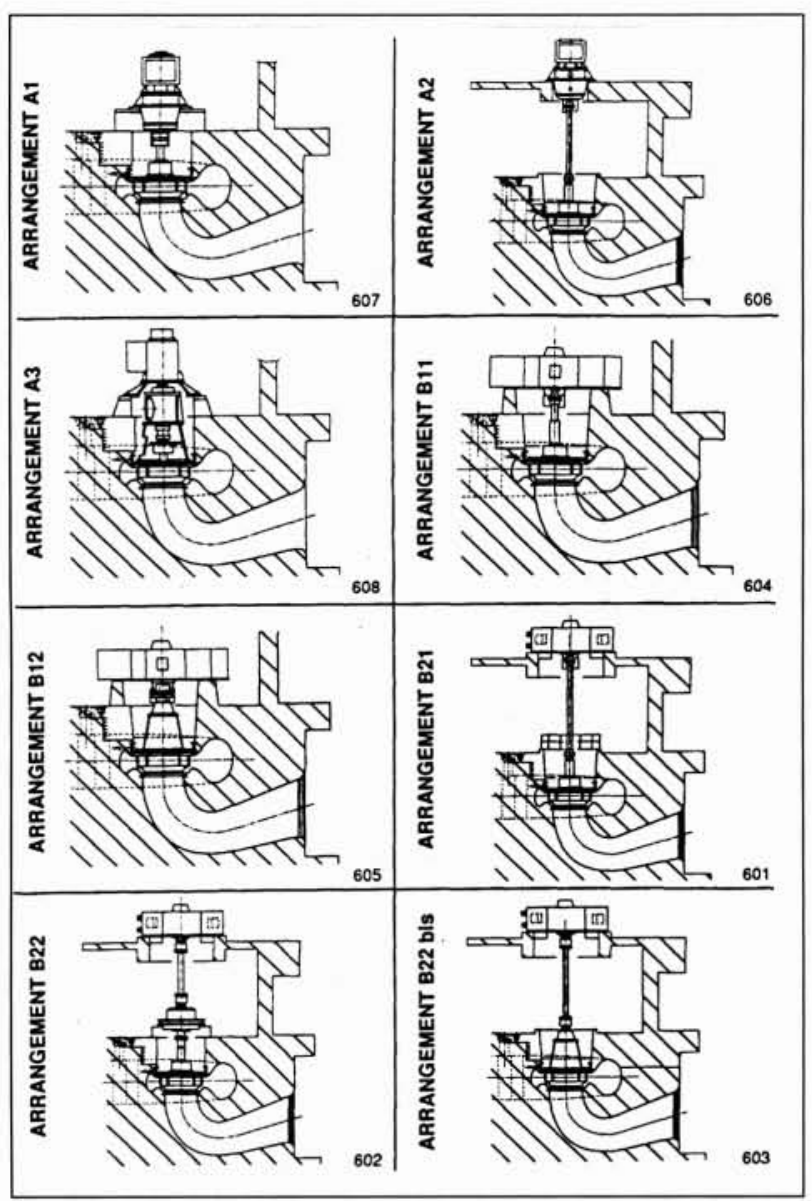

7. Différents arrangements possibles à base de pompes.
En fonction des choix technico-économiques du type d'entraînement, de nombreuses possibilités d'aménagement existent comme le montre la figure 7. La technologie de la pompe en est influencée dans la mesure où la poussée axiale peut être reprise par une butée dans la pompe ou dans le réducteur et/ou le moteur.

Là encore, ce sont des considérations technico-économiques et des problèmes de maintenance qui influencent les choix.

\section{IV — CHOIX DES MATÉRIAUX, MAINTE- NANCE, FIABILITÉ}

Les avantages essentiels de la pompe à volute en béton sont liés au fait que d'une part, le conduit et la volute sont en béton et résistent donc parfaitement aux problèmes de corrosion et d'abrasion particulièrement en eau de mer, les durées des bétons étant supérieures à 40 ans sans intervention. D'autre part, la quantité de béton entourant les conduits d'aspiration et les volutes réduit les bruits et vibrations et facilite le supportage des masses importantes de l'entraînement. Là encore, la fiabilité d'ensemble du pompage en est améliorée.

Le nombre faible de pièces en contact avec l'eau (roue, pièce scellée, l'arbre étant protégé par une chemise) limite les risques d'érosion et de corrosion. L'expérience acquise sur des centaines de pompes en fonctionnement, permet de proposer le type de matériau adéquat pour résister aux agressions plus ou moins importantes du fluide pompé.

La maintenance est réduite eu égard au faible nombre de pièces et par la présence d'un palier à huile, à cuve tournante auto-lubrifiée, hors d'eau.

Les grandes visites peuvent être espacées de 5 à 7 ans avec des durées de fonctionnement supérieures à $50000 \mathrm{~h}$. Une surveillance annuelle des principaux jeux des pièces d'usures suffit au titre de la maintenance préventive. De ce fait, le taux de disponibilité de ce matériel est exceptionnel, ce qui justifie pleinement son utilisation pour les circuits de refroidissement des grandes centrales thermiques et nucléaires.

\section{V — RÉCENTS DÉVELOPPEMENTS}

Le développement de la demande pour des pompes avec des hauteurs inférieures à $12 \mathrm{~m}$ conduit à rechercher les solutions les plus compactes possibles tout en conservant des performances (rendement, forme des caractéristiques, NPSH) aussi élevées que possible.

Des pompes à volute béton de vitesse spécifique élevée $N s>130$ ont été développées

$$
\mathrm{Ns}=\frac{N(\mathrm{rpm}) \times Q^{0.5}\left(\mathrm{~m}^{3} / \mathrm{s}\right)}{H^{0.75}(\mathrm{~m})}
$$

Ces nouvelles pompes doivent, en outre, offrir une simplicité de forme des volutes et des coudes d'aspiration pour minimiser les coûts des coffrages et faciliter la mise en place du ferraillage et du béton.

Le type de pompe à grande vitesse spécifique impose l'utilisation de roues hélico-centrifuges qui génèrent à leur tour de nouvelles contraintes : 


$$
\begin{gathered}
\frac{\mathrm{H} \mathrm{Q}=0}{\mathrm{H} \text { nominal }} \geq 2 \\
\frac{\text { Puissance } \mathrm{Q}=0}{\text { Pnominal }} \geq 1,5
\end{gathered}
$$

- Augmentation de la longueur du porte-à-faux palier-bague d'aspiration par rapport à une solution centrifuge.

Cette dernière contrainte génère une flèche de l'arbre, sous l'effet de la poussée radiale, qui est alors plus élevée que dans une solution centrifuge. Cela n'est pas sans conséquence sur le dimensionnement mécanique des composants de la pompe : palier, diamètre d'arbre, jeu radial aux bagues.

Il est donc nécessaire de faire les développements indispensables pour résoudre ces nouveaux problèmes et optimiser la conception. Parmi les actions menées à ce titre, on peut citer :

\section{- Au plan hydraulique}

La simplification et la compacité des formes des conduits hydrauliques (volute et coude d'aspiration) ne doivent pas engendrer de pertes de rendement supplémentaires. C'est pour cette raison que des calculs d'optimisation et de validation des simplifications ont été menées à l'aide de code de calculs d'écoulement du type NavierStokes, seuls aujourd'hui aptes à prendre en compte les écoulements secondaires et les zones décollées dans la volute. Pour ce faire, les conditions d'entrée de l'écoulement dans la volute sont imposées en se basant sur les sondages effectués lors d'essais sur modèle réduit. L'analyse est faite dans des plans radiaux et dans la tubulure de refoulement de la volute en termes de champs de pression, de vitesse et d'écoulement secondaires (fig. 8).

\section{- Au plan mécanique :}

Comme indiqué précédemment, la connaissance de la flèche est un élément fondamental pour bien dimensionner les composants de la pompe (arbre, palier, etc ...). La poussée radiale exercée par l'écoulement sur la roue doit être déterminée avec précision dans toute la zone d'utilisation de la pompe $y$ compris dans les phases transitoires de Démarrage-Arrêt. A cette fin, les modèles réduits des pompes à volute sont équipés d'un dispositif de mesure de poussées radiales. Il s'agit d'un palier hydrostatique de grande raideur. $\mathrm{La}$ poussée radiale est déduite de l'intégration des pressions moyennes mesurées dans chacune des 4 alvéoles du palier. On étalonne le dispositif en appliquant des efforts de direction et module variés. La figure 9 donne, en valeurs relatives, les coefficients de Stepanoff recalculés à partir des efforts radiaux moyens mesurés sur une pompe de vitesse spécifique élevée 130.

$\mathrm{K}$ coefficient de Stepanoff $=\frac{\text { Poussée Radiale }(\mathrm{daN})}{\mathrm{b}_{(\mathrm{mm})} \cdot \text { Déq }_{(\mathrm{mm})} \cdot \mathrm{H}_{(\mathrm{m})} \cdot 10^{-3}}$
$\begin{aligned} & \text { avec : }\end{aligned}$

$\mathrm{b}=$ hauteur de la roue

Déq = diamètre équivalent de sortie roue

$\mathrm{H}=$ hauteur manométrique totale pompe

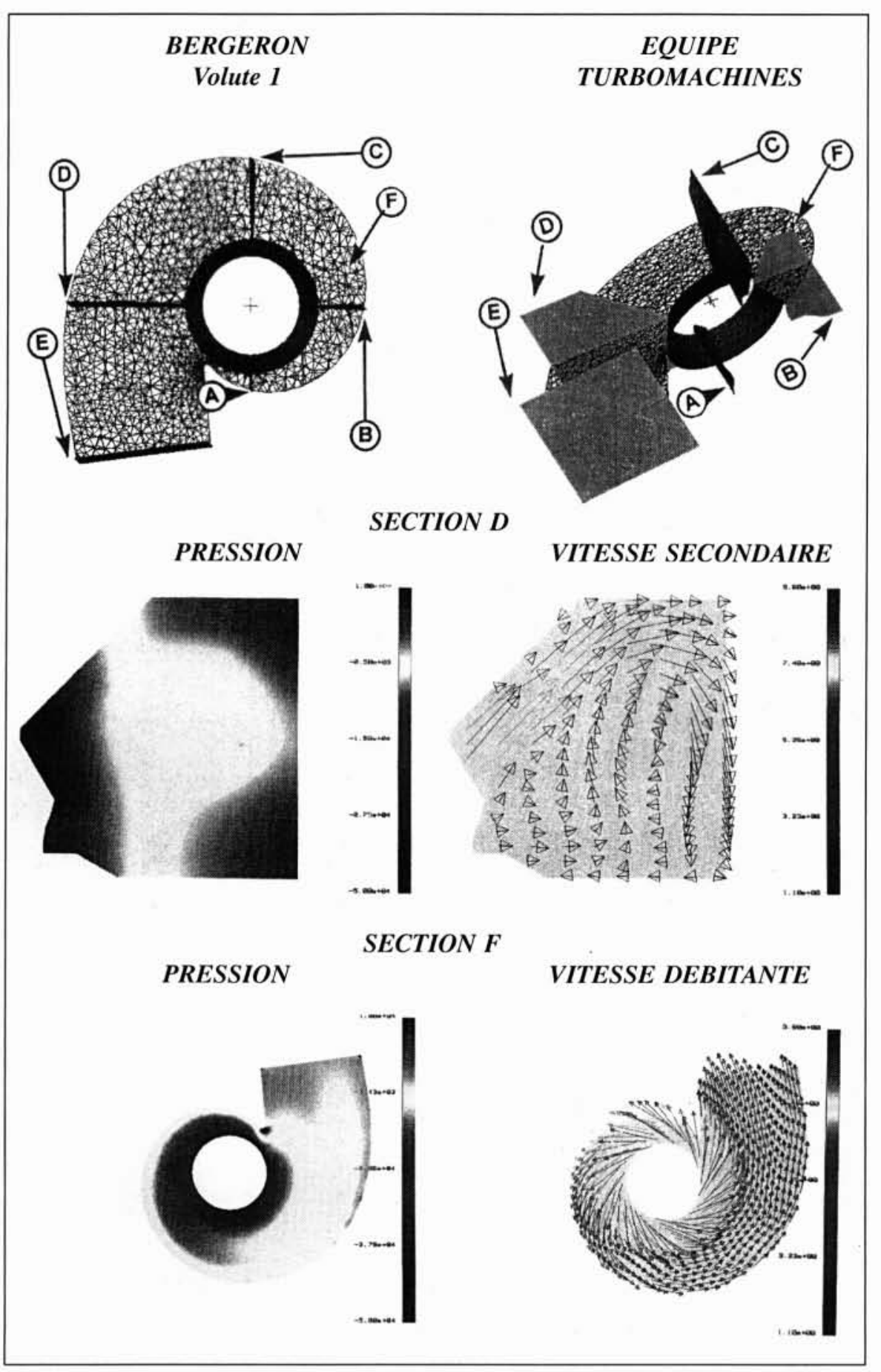

8. Modélisation des champs de pression, vitesse et écoulement secondaires. 


\section{Applications industrielles de la mécanique des fluides}

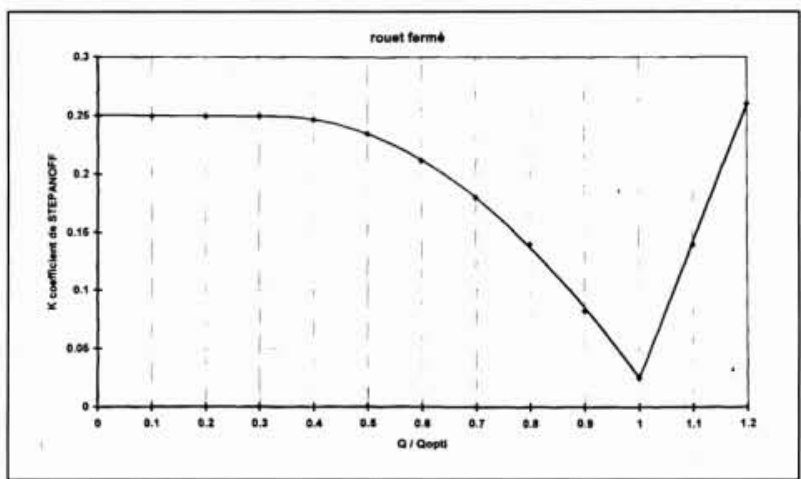

\section{CONCLUSION}

La fiabilité des pompes à volute en béton, leur faible coût de maintenance et leurs performances élevées en font des systèmes d'une grande souplesse pour assurer des grands transferts tant en eau douce qu'en eau de mer. Des développements continus sont entrepris pour améliorer les performances, diminuer les coûts tout en conservant le même taux de fiabilité.

9. Coefficient de Stepanoff de pompe à volute rapide. 